\title{
Efficient Cluster Head Selection Scheme for Wireless Sensor Network Using Deterministic Protocol
}

\author{
Sandeep Singh Meelu ${ }^{1}$, Mamta Katiyar ${ }^{2}$ \\ I'Student, Department of Electronics and Communication Engineering Maharishi Markandeshwar \\ University, Mullana (Ambala), Haryana, India) \\ ${ }_{2}^{2}$ (Guide, Departments of Electronics and Communication Engineering Maharishi Markandeshwar \\ University, Mullana (Ambala), Haryana, India)
}

\begin{abstract}
The Wireless Sensor Networks are emerging Technology of 21st Century and It is estimated that by the coming years more and more wireless sensors will be deployed for applications as diverse as environmental monitoring, agricultural monitoring, machine health monitoring, surveillance, and medical monitoring. These networks, which connect the physical world with the digital world, provide us with a richer understanding of our environment and with the ability to more accurately control our surroundings. However, there are many challenges that must be addressed before the full potential of these networks are realized. In this paper we introduced improved DEC (I-DEC) protocol which is capable of improving stability as well as lifetime of the WSN network over other existing protocols. An overview of existing protocols with their advantages, limitations and existing improvements is also presented in this paper. The results obtained from proposed DEC shows better performance with respect to energy consumption over existing protocols.
\end{abstract}

Keywords: Cluster Head Selection, Clustering Protocol, Deterministic Clustering, Energy Efficient, WSN lifetime.

\section{Introduction}

A wireless sensor network consists of tiny devices called sensor nodes which are deployed over a geographical area for monitoring physical phenomena like temperature, humidity, vibrations, and so on [1]. A sensor node consists of three basic units: a sensing unit for collecting data from surrounding environment, a computing unit for data processing and storage, and a wireless communication unit for transmission of data. A power source is also required by a sensing node to perform the task. The power source used by sensor node is a battery with limited energy and also it is not possible to recharge or replace the battery because nodes may be deployed in unpractical environment and thus the sensor network must have enough lifetime to fulfill the requirements of the application. The wireless sensing technologies offer vast opportunities for research and developments.

The wireless sensing technologies offer vast opportunities for research and developments. The Wireless Sensor Networks (WSN) are one of the most significant technologies in 21 st century. WSN can operate in a wide range of environments and thus can be used for various applications:

The development of WSN applications in agriculture and food industry has attracted considerable research efforts in recent years because WSNs are very suitable for distributed data collecting in tough environments such as greenhouses, warehouses, croplands and also can be used in environmental monitoring so that the variability present in the environment can be detected, for example alerting farmers at the onset of frost damage [2]. Wireless sensor technology also has a great potential for industrial and commercial applications as this technology can monitor data such as pressure, humidity, temperature, flow, level, viscosity, density and vibration intensity measurements can be collected through sensor nodes and transmits to control system for further operation wirelessly for industrial applications like machine health monitoring, structural heath monitoring [3].

Sometimes it is impossible to install equipment in some areas because of some reasons like: lack of access to power or unable to make a wired connection. Due to these problems WSN is used. The advantages of using WSN are: no need for hardwiring, low distance limits, high energy and cost efficiency. These advantages of WSNs are taken to use them in weather monitoring stations to monitor weather conditions by detecting changes in environmental parameters such as humidity, temperature, and so on. Disaster alarm systems can also take help from WSNs as these can be used to detect Landslides by detecting movements in soil, forest fire detection by measuring gases produced during fire, and so on [4].

As mentioned above a Wireless Sensor Network (WSN) consists of number of sensor nodes. These sensor nodes are scattered in an environment called sensor field. The basic communication architecture for WSN is shown by Fig.1. The sensing nodes communicate with sink via multiple hops. The sink communicates with user with help of internet or satellite networks [5]. 


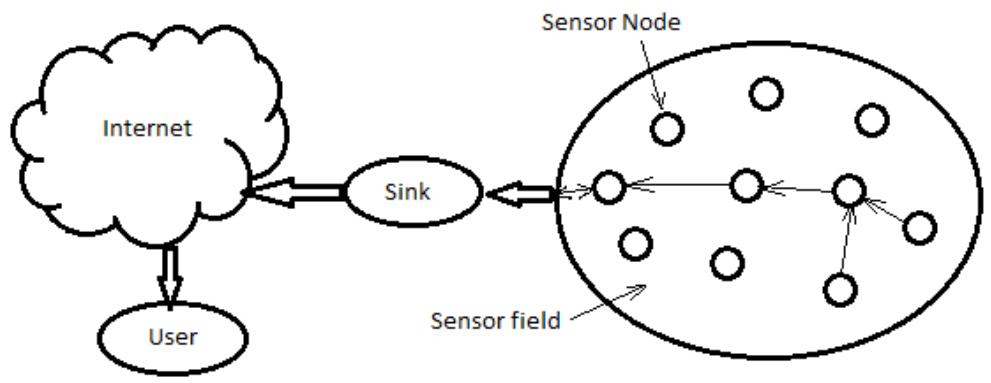

Fig. 1 Basic Architecture of WSN

\section{Wsn Network Models}

The routing protocols in WSNs can be divided into two categories: flat routing and clustering routing and this classification is based on network structure. In flat routing all nodes have same functionalities and they perform same tasks and the data transmission is done by multiple hops. On other hand, in a clustering or hierarchical protocol, nodes have different tasks and they are divided into groups called clusters [6].

In each cluster there is a cluster head $(\mathrm{CH})$ which communicates with base station (BS), and other nodes called member nodes which collect and send data to the cluster head [7]. The selection scheme for cluster head must be energy efficient and it must also be capable of maintaining overall stability of network. The authors in [8] [9] [10] [11] [12] [13] used have used clustering to manage WSNs. However, the authors in [8] [9] [10] [11] [12] have used probabilistic-based model to manage energy consumption in WSNs. One of the goals of these protocols is to extend the WSN lifetime without considering the local information i.e. the residual energy of each node. The downside of such protocols is that there is no guarantee that the desired number of cluster heads (CHs) will be elected or the elected $\mathrm{CH}$ will have enough energy to perform its duty as a leader and author in [13] used a deterministic cluster head selection algorithm.

Low Energy Adaptive Clustering Hierarchy (LEACH) is one of the mostly used hierarchical clusterbased routing protocols for wireless sensor network [8]. In LEACH as mentioned above the network is divided into number of clusters which uses TDMA (Time Division Multiple Access) schedule for member nodes. There are some problems associated with LEACH Protocol: The cluster head is selected randomly so each node in cluster has same probability to become cluster head. After numerous rounds, the node with high remaining energy and node with low remaining energy have same probability of becoming cluster head. If the node with low remaining energy is chosen as cluster head, it will run out of energy and die quickly which affects network lifetime. The division of clusters is also done in random fashion which results in uneven distribution of clusters. The divided clusters have non uniformities like: one cluster has a lot more nodes than other cluster and some cluster heads are at centre and some are at edge of cluster far from other members. These non uniformities affect the performance of network.

Stable Election Protocol (SEP) is also a further modification to the LEACH protocol. In this protocol two types of nodes and two level hierarchies are considered. It is based on weighted electron probabilities of each node to become cluster head according to remaining energy in each node. SEP is a heterogeneous aware protocol and it prolongs the time interval before the death of first node which is referred as stability period [9]. In order to prolong stable region, SEP maintain a well balance of energy consumption. The advanced nodes have to become cluster heads earlier than normal nodes. The cluster head election is arbitrarily selected and distributed based on the fraction of energy of every node assuring a uniform use of the nodes energy. The main limitation of SEP is that election of cluster heads among the two type of nodes is not dynamic, which results that the nodes that are far away from the powerful nodes will die first. The improvement to SEP protocol is also introduced in [10].

Hybrid Energy Efficient Distributed Clustering (HEED) protocol is a extension of LEACH protocol, in this protocol the residual energy is used as primary parameter while other topology features like node degree, distances to neighbours are used as secondary parameter to attain power balancing in network. The clustering process is split into number of iterations, in every iteration nodes that are not covered by any cluster head and thus doubles their probability of becoming a cluster head. As these energy-efficient clustering protocols further enables each node to probabilistically and independently decide its role in the clustered network. Moreover they cannot guarantee optimal elected set of cluster heads [11]. This protocols introduced uniform Cluster head distribution across the network by enabling low power levels of cluster endorse an increase in spatial reuse while high power levels of clusters are needed for intercluster communication. The long range communication 
from $\mathrm{CHs}$ to sink is possible as communication between $\mathrm{CHs}$ and $\mathrm{BS}$ provides more energy conservation. The main limitation of HEED is that it imposes significant overhead in network which causes remarkable energy dissipation which results in reduced network lifetime. The CHs near the sink might die earlier because of high workload on them and also the uncertain $\mathrm{CHs}$ that do not become final $\mathrm{CHs}$ leave some uncovered nodes, these nodes are forced to become $\mathrm{CH}$ and these forced $\mathrm{CH}$ may not have any member associated between them. As result, unexpected number of $\mathrm{CHs}$ are generated which make the unbalance in energy consumption of network.

Power Efficient Gathering in Sensor Information System (PEGASIS) is an improved version of LEACH protocol [12]. This protocol is guaranteed by two characteristics, only one node communicates with base station at a time and rest of the nodes communicates only with their neighbours. Each node communicates with the nearest neighbour by adjusting its power signal strength. In this way, each node measure the distance to the neighbourhood nodes in order to find the nearest node. In this process a chain is formed and a leader is elected from chain on the basis of residual energy in every round. The leader collects data from neighbours and transmits the collected data to base station and thus the average energy consume by each node per round is decreased. This approach also reduces the bandwidth requirement by lowering the overhead required. The PEGASIS also has limitations for example: it is complicated task for all nodes to maintain a complete database about the location of all other nodes within the network and also the communication in PEGASIS suffers from excessive delays caused by single chain for distant nodes and a high probability for any node to become a bottleneck.

It is worth to note in [13] that a deterministic cluster head selection algorithm can outperform a probabilistic-based algorithm in terms of energy consumption. Deterministic Energy-efficient Clustering (DEC) protocol is a purely deterministic model that utilizes clustering to organize WSN. This protocol is dynamic, distributed, self-organizing and more energy efficient than existing protocols. The approach of this protocol is simple that is to minimize computational overhead cost to self-organize the network. This protocol has better performance with respect to energy consumption in both heterogeneous and homogeneous network. This protocol uses residual energy of each node in the cluster for election process of $\mathrm{CH}$. The uncertainties in the cluster-head elections have been minimized in DEC. The setup phase used in LEACH is modified, but the steady-state phase is kept same as that of in LEACH protocol. Since node's energy can be determined a priori, the $\mathrm{CH}$ election process is reorganized by using the RE of each node. In DEC, the BS elects no. of cluster-heads at round $\mathrm{m}$ for the network. The BS can only take part in the election of $\mathrm{CHs}$ if and only if $\mathrm{m}=1$. The elected CHs advertise their role using CSMA MAC just as in LEACH. However, in DEC unlike in LEACH, the join request message will contain CM-ID (Cluster member ID), CH-ID (Cluster Head ID) and CM-RE (cluster member-residual energy) and the header that indicates it as a request. This way the RE information of CMs is known to their respective $\mathrm{CHs}$ thus localized and it can be utilized for $\mathrm{CH}$ rotation in the subsequent rounds.

However, there are some problems associated with DEC protocol in [13] if the number of rounds for DEC protocol would increased then the nodes deployed in the network become dead earlier than LEACH or SEP protocol. Therefore, In DEC protocol it is difficult to maintain a balance between maximum number of rounds a network survive and stability of network. It is difficult to achieve stability of network with the high network lifetime.

The author in [14] proposed Two Phase Clustering Method for cluster head selection has also been done in order to improve the LEACH protocol in [8]. In this research, a two stage clustering protocol based on Self Organizing Map (SOM) neutral network and Modified Fuzzy Probabilistic Clustering Algorithm (MFPCM) with the purpose of balancing the energy consumption. The clustering is based on two important conditions, coordinates of sensor nodes and energy level. Consequently, this two stage clustering method can prevent from premature death of the nodes and permit for random death of them.

The heterogeneous HEED (H-HEED) is proposed in [15], this protocol basically used in heterogeneous WSNs and it adds extra functionality to HEED protocol in [11]. The H-HEED also serves same purpose which is main requirement of WSNs, to prolong network lifetime and energy efficiency. The simulation results shows that the throughput and packet delivery ratio are improved by using H-HEED and the delay and energy consumption is also less than HEED.

Recent study by author in [16] proposed the Hop PEGASIS approach which is more efficient than the PEGASIS proposed in [12]. If there is a direct transmission between CHs and base station, the cluster head that is situated far away from the base station uses strong signals while transmission to the base station thus leads to more energy consumption, so in this protocol the lifetime of network gets improved. The experimental results shows that, in hop PEGASIS the nodes survived around 3000 rounds better than LEACH (1100 rounds) as well as PEGASIS (2000 rounds). 


\section{Proposed Improved Dec Protocol}

The improved DEC (I-DEC) protocol is proposed in which the number of rounds as well as stability of network is increased as compared to original DEC protocol [13]. The ideas behind proposed DEC protocol are given below:

In proposed DEC the multilevel clustering is employed in which four levels of nodes are defined which is better suited for defining heterogeneous environment as compared to three levels of nodes multilevel clustering model define in the original DEC protocol. It must be noted that the total energy of network is kept same as that of original DEC protocol. This new clustering model helps in increasing the network stability in heterogeneous environment.

The distance between member nodes and nearby cluster heads is calculated, after calculation distance a comparison is made that ensures the minimum distance between the member node and the cluster head and then the join request message is only send to that cluster head which fulfill the condition of minimum routing distance. In this way the energy consumption via transmission of data from member node to $\mathrm{CH}$ is reduced to possible extent which results in improve network lifetime. So, the number of rounds gets increased to possible extent without affecting the network stability.

The proposed DEC ensures that selected cluster head is different at different round. After completion of first round the previously selected cluster head is released from the role of cluster head without checking its residual energy. Instead of checking residual energy of previously selected cluster head the proposed DEC checks for the node which has maximum residual energy in whole cluster so that it becomes new cluster head for next round. This cycle repeat itself for the defined number of rounds. This method guarantees the uniform energy consumption of the network by selecting different cluster heads at different rounds thus the stability gets increased to possible extent without affecting the number of rounds. The initial parameters taken into consideration are shown in Table 1.

Table 1 Initial parameters for implementing the DEC protocol.

\begin{tabular}{|l|l|}
\hline Description & Value \\
\hline Dimensions of Field & $100 \mathrm{~m} \mathrm{x} \mathrm{100m}$ \\
\hline No of Nodes & 100 \\
\hline Max no of Rounds & 4000 \\
\hline Probability of a node to become CH & 0.1 \\
\hline Initial energy of each node & $0.5 \mathrm{~J}$ \\
\hline Transmission energy of node & $50^{*} 0.000000001 \mathrm{~J}$ \\
\hline Receiving energy of node & $50^{*} 0.000000001 \mathrm{~J}$ \\
\hline Data aggregation energy & $5 * 0.000000001 \mathrm{~J}$ \\
\hline Energy dissipation for free space & $10^{*} 0.000000000001 \mathrm{~J}$ \\
\hline Energy dissipation for multi-path delay & $0.0013 * 0.000000000001 \mathrm{~J}$ \\
\hline Packet size & 4000 \\
\hline
\end{tabular}

The proposed DEC protocol will ensure different Cluster head selection at different rounds as explained in above discussion.

At first, the base station (BS) selects $\mathrm{n}$ number of cluster heads at round $\mathrm{r}=1$ and initially the new cluster head check loop says yes and the cluster head ID (CH-ID) is broadcasted and $\mathrm{CH}$ waits for the request from the cluster members. The cluster members send join request message accordingly to minimum routing distance to the nearest cluster head. The cluster head stop waiting and setup a TDMA schedule for all cluster members (CMs). $\mathrm{CH}$ then checks residual energies (REs) of all of its CMs. The CMs then start transmission of data to the $\mathrm{CH}$ which after receiving aggregates the data and transmits to base station then the election process for another cluster head selection is initiated.

In election process the previously selected cluster head is released from its role as cluster head and a new node which having highest energy in the whole cluster is selected as new cluster head and then again loop starts from check new cluster head and the above explained procedure run again.

\section{Results And Comparisons}

Proposed DEC (I-DEC) protocol is verified using simulations and its performance is compared with existing protocols i.e. LEACH, E-SEP and existing DEC.

The Figure 2 showing alive nodes versus number of rounds plot for original DEC protocol. The FND (First Node Dead) also known as stability period is at $1839^{\text {th }}$ round means that the network is stable up to 1839 rounds. The LND (Last Node Dead) also known as instability period is at $2350^{\text {th }}$ round also it shows that network lifetime is about 2350 rounds by using original DEC protocol. The proposed DEC also performs better than other protocols. The packets sends to base station are stable as compared to ESEP and LEACH and also duration of stable rate of sending packets is 2400 rounds which is better than original DEC protocol. Figure 3 shows that the proposed DEC protocol is around 1450 rounds stable then existing LEACH protocol as well as 
lifetime of network is also around 1500 rounds more than that of LEACH protocol. The Enhanced SEP (ESEP) protocol has however extend the network lifetime but in terms of stability the Proposed DEC is around 950 rounds more stable than ESEP protocol.

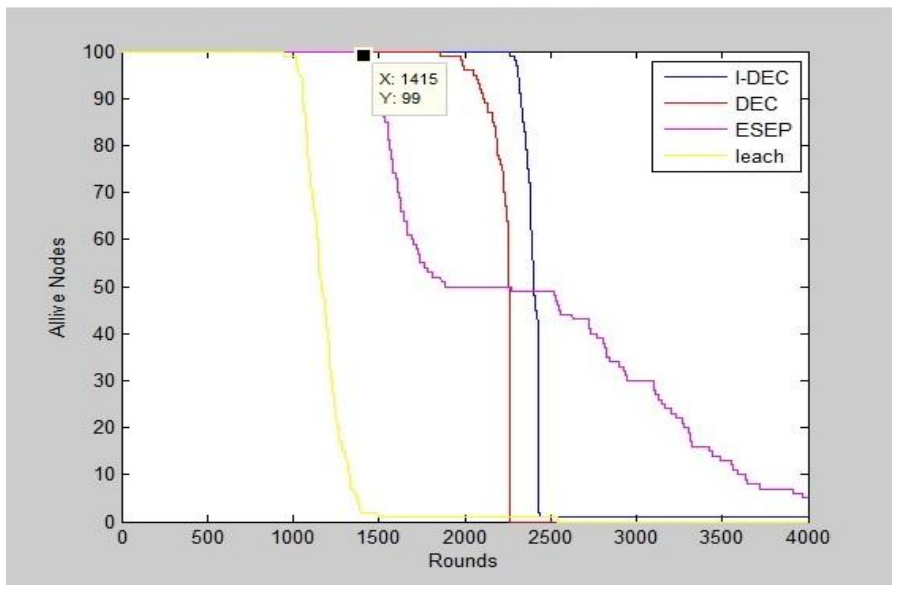

Fig. 2 Alive nodes v/s rounds plot comparison of proposed DEC with other existing protocols.

The energy management of proposed DEC is better than Original DEC as shown in Figure 3 proving that proposed DEC is more energy efficient than original DEC protocol. Figure 3 shows comparison between original DEC and proposed DEC in terms of total energy versus number of rounds.

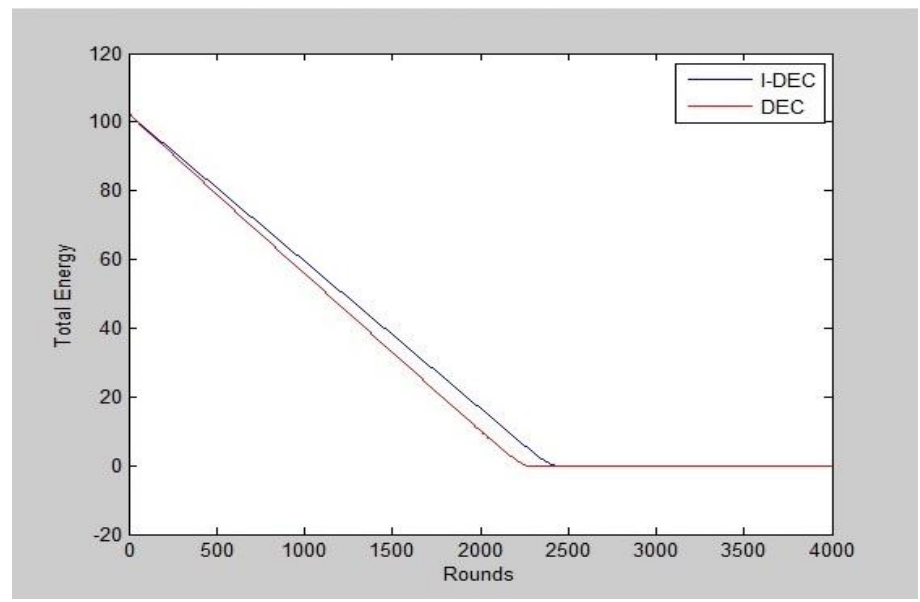

Fig. 3 Comparison between original DEC and proposed DEC

In Figure 4 the Dead nodes with respect to number of rounds is shown and here again the proposed DEC perform better than other protocols.

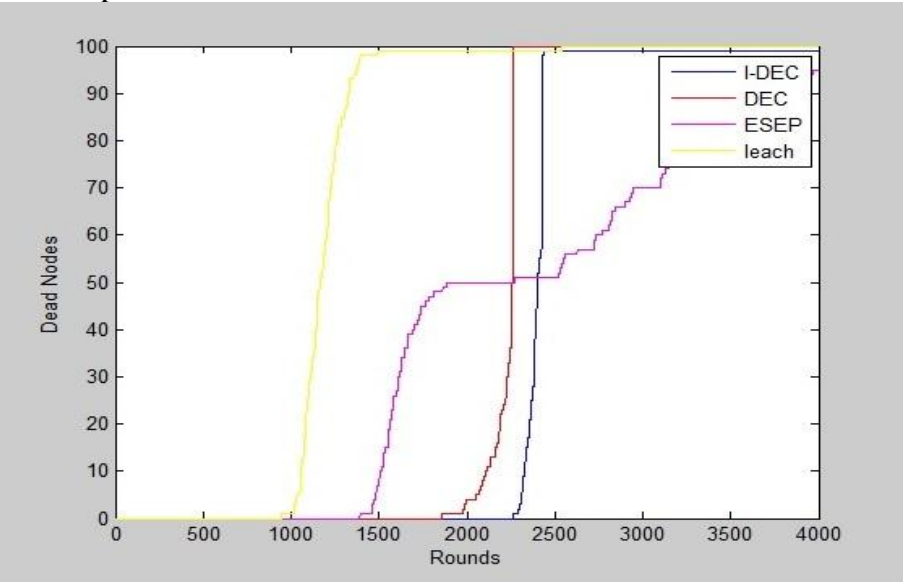

Fig. 4 Dead nodes v/s rounds plot comparison of proposed DEC with other existing protocols. 
Table 2 comparison of existing protocols with the proposed DEC protocol.

\begin{tabular}{|c|c|c|}
\hline Protocol & First Node Dead & Last Node Dead \\
\hline LEACH & 900 & 1500 \\
\hline E-SEP & 1400 & 4000 \\
\hline DEC & 1839 & 2350 \\
\hline Proposed I-DEC & 2350 & 2400 \\
\hline
\end{tabular}

Table 2 shows comparison of existing LEACH, ESEP, DEC protocols with the proposed DEC protocol. It can be seen from the comparison that proposed DEC performed better than other existing protocols in most of aspects.

\section{Conclusion}

Recently, there has been an increase in the use of wireless sensor networks for monitoring environmental information (temperature, sound levels, humidity etc) across an entire physical space. The main challenging task in this network is lifetime with stability. There are different types of protocols in WSN used to increase network lifetime but each protocol address one problem by leaving other. In this paper, an improved version of DEC protocol is developed which is capable of balancing stability with the network lifetime and also has better energy management then existing DEC protocol.

\section{References}

[1]. Giuseppe Anastasi, Marco Conti, Mario Di Francesco, Andrea Passarella, Energy conservation in wireless sensor networks: A survey, Computer Networks, Elsevier, Volume 7, Issue 3, Pages 537-568, May 2009.

[2]. Luis Ruiz-Garcia, Loredana Lunadei, Pilar Barreiro and Jose Ignacio Robla, A Review of Wireless Sensor Technologies and Applications in Agriculture and Food Industry: State of the Art and Current Trends, Sensors, Volume 9, Issue 6, June 2009.

[3]. Gang Zhao, Davis, Wireless Sensor Networks for Industrial Process Monitoring and Control: A Survey, Macrothink Institute, Network Protocols and Algorithms, ISSN 1943-3581, Vol. 3, No. 1, 2011.

[4]. Cholatip Yawut and Sathapath Kilaso, A Wireless Sensor Network for Weather and Disaster Alarm Systems, International Conference on Information and Electronics Engineering, IPCSIT IACSIT Press, Singapore, vol.6 2011

[5]. Mr. Puneet Garg, Mr. Kuntal Saroha, Mrs. Ruchika Lochab, Review of Wireless Sensor Networks -Architecture and Applications, IJCSMS International Journal of Computer Science \& Management Studies, Vol-11, Issue 01, May 2011.

[6]. Md. Habibe Azam1, Abdullah-Al-Nahid,Md. Abdul Alim, Md. Ziaul Amin, A Survey and Comparison of Various Routing Protocols of Wireless Sensor Network (WSN) and a Proposed New TTDD Protocol Based on LEACH, International Journal of Computer and Network Security, Vol. 2, No. 8, August 2010.

[7]. Kiran Maraiya, Kamal Kant, Nitin Gupta, Efficient Cluster Head Selection Scheme for Data Aggregation in Wireless Sensor Network, International Journal of Computer Applications (0975 - 8887) Volume 23- No.9, June 2011.

[8]. Wendi Rabiner Heinzelman, Anantha Chandrakasan,and Hari Balakrishnan, Energy-Efficient Communication Protocol for Wireless Microsensor Networks, 33rd Hawaii International Conference on System Sciences, IEEE, 2000.

[9]. G. Smaragdakis, I. Matta, A. Bestavros, SEP: A Stable Election Protocol for clustered heterogeneous wireless sensor networks, In Second International Workshop on Sensor and Actor Network Protocols and Applications (SANPA), 2004

[10]. F. A. Aderohunmu, J. D. Deng, and M. K. Purvis, Enhancing Clustering in Wireless Sensor Networks with Energy Heterogeneity, International Journal of Business Data Communication and Networking, 2011

[11]. O. Younis and S. Fahmy, HEED: A Hybrid, Energy-Efficient, Distributed Clustering Approach for Ad Hoc Sensor Networks, IEEE Transactions on Mobile Computing, 3:366-379, 2004.

[12]. S. Lindsey, C. Raghavendra, PEGASIS: Power Efficient Gathering in Sensor Information Systems, IEEE Aerospace Conference Proceedings, Vol. 3, 9-16, pp.11251130, 2002.

[13]. Femi A. Aderohunmu, Jeremiah D. Deng, Martin K. Purvis, A deterministic Energy-efficient Clustering Protocol for Wireless sensor network, Information Science Department, University of Otago Dunedin, New Zealand, pages 341 - 346 Sensor Networks and Information Processing (ISSNIP), Dec. 2011

[14]. Rathi and Viswanathan, Two Phase Clustering Method for Leach Protocol For Effective Cluster Head Selection, Journal of Computer Science, Science Publications 2014.

[15]. Sasikumar M, Dr. R. Anitha, Performance Evaluation of Heterogeneous-HEED Protocol for Wireless Sensor Networks, International Journal of Advanced Research in Computer and Communication Engineering (IJARCCE), Vol. 3, Issue 2, February 2014.

[16]. Laiali Almazaydeh, Eman Abdelfattah, Manal Al- Bzoor, and Amer Al- Rahayfeh, Performance Evaluation of Routing Protocols in Wireless Sensor Networks, International Journal of Computer Science and Information Technology, Volume 2, Number 2, April 2010 . 